\title{
Virtuelle Autopsie (Virtopsy) in der Forensik
}

Thali, Michael J

\begin{abstract}
The aim of "virtopsy" is to utilize modern imaging technology to optimize classical autopsy documentation. The benefits of virtopsy include examiner-independent, objective 3D documentation and its non-invasive approach. Virtopsy is an option in cases where autopsy is rejected by family members or for religious reasons. It is also suitable as a rapid identification and examination tool in large-scale disasters. Forensic findings can be presented to prosecutors and courts in 3D and without bloody images.
\end{abstract}

DOI: https://doi.org/10.1007/s00292-011-1520-5

Other titles: Virtual autopsy (virtopsy) in forensic science: from the scalpel to the scanner

Posted at the Zurich Open Repository and Archive, University of Zurich

ZORA URL: https://doi.org/10.5167/uzh-85289

Journal Article

Published Version

Originally published at:

Thali, Michael J (2011). Virtuelle Autopsie (Virtopsy) in der Forensik. Der Pathologe, 32 Suppl:292-295. DOI: https://doi.org/10.1007/s00292-011-1520-5 
Pathologe 2011 - [Suppl 2] 32:292-295

DOI 10.1007/s00292-011-1520-5

C) Der Verfasser 2011

\section{Thali}

Institut für Rechtsmedizin, Universität Zürich, Schweiz

\section{Virtuelle Autopsie (Virtopsy) in der Forensik}

\section{Vom Skalpell zum Scanner}

\section{CSI prägt die Rechtsmedizin in der öffentlichen Wahrnehmung}

Die Rechtsmedizin kennt man nicht mehr von klassischen Krimisendungen wie seinerzeit „Colombo“, sondern von „CSI“ (Crime Scene Investigation). Diese amerikanische Fernseherfolgsserie gibt es mittlerweile als Krimivariation aus verschiedenen US-Städten. Unter den CSI-Sendungen wird die gesamte Forensik zusammengefasst. Hochattraktive Personen lösen innerhalb weniger als einer Stunde hochkomplexe Fälle. Diese Sendungen haben die Forensik medial in ein völlig anderes Licht gestellt. Vor Jahren galt die Gerichtsmedizin als etwas sehr Exotisches und war im Fernsehen kaum präsentierbar. Mit den CSI-Sendungen hat sich die Forensik in das allabendliche Programm der fernsehwilligen Zuschauer gedrängt und erfreut sich sehr hoher Einschaltquoten.

Die gerichtsmedizinischen Institute haben sich über die Jahre zu forensischen Instituten gewandelt, mit zusätzlichen $\mathrm{Ab}$ teilungen der forensischen Pharmakologie bzw. Toxikologie, forensischen Molekularbiologie sowie der Verkehrsmedizin.

Die bahnbrechenden Erfolge der „Gerichtsmedizin“ sind zweifelsohne im letzten Jahrhundert im Bereich der Labormedizin geschehen. Toxikologisch können immer feinere Stoffe analysiert werden. Die moderne Forensik wäre ohne DNA-Technologie, die auch feinste Spuren den Tätern zuordnen kann, undenkbar. Um die eigentliche „Leichengerichtsmedizin“ war es lange sehr ruhig. Mit klassischen Methoden wurden über Jahrhun- derte Leichen aufgeschnitten und forensisch relevante Todesursachen eruiert. Obduktionsmethoden wurden über die Jahre zunehmend verfeinert und für Spezialfragen optimiert. Vorreiterrolle war hier der europäische Raum mit den Wurzeln in der ungarisch-österreichischen Gerichtsmedizin. Die Rechtsmedizin blieb im Kerngebiet wesentlich der Leiche treu. Teilweise gab es an einzelnen Instituten die Erweiterung in die sogenannte klinische Rechtsmedizin, bei der zunehmend auch lebende Opfer von Gewalteinwirkungen rechtsmedizinisch untersucht werden.

Wesentliche Aufgabe der Gerichtsmedizin für die Gesellschaft ist und bleibt es, Tötungsdelikte zu erkennen und forensische Fragestellungen, hauptsächlich für die Strafverfolgungsbehörden, aber auch andere Interessengruppen wie Versicherungen etc., zu detektieren. So soll vermieden werden, dass Tötungsdelikte überse- hen werden. Der ehemalige Präsident des Bundeskriminalamts, Horst Herold, soll einmal gesagt haben: „Wenn auf jedem Grab eines Ermordeten, von dem wir irrtümlich annehmen, dass er eines natürlichen Todes gestorben sei, eine Kerze brennen würde, wären nachts alle Friedhöfe hell erleuchtet“. Man könnte auch modifiziert postulieren: Sage mir, wie die Forensik in Deiner Gesellschaft ist, und ich sage Dir, in welcher Gesellschaft Du lebst.

Die beste Definition des sogenannten außergewöhnlichen Todesfalls, $d$. h. jene Fälle, die unter forensischen Gesichtspunkten weiter untersucht werden, also in den Fokus der forensischen Betrachtung rücken sollten, stellt immer noch die von Prof. Fritz Schwarz nach seiner Emeritierung in Zürich um 1970 niedergeschriebene Definition dar:

Ein außergewöhnlicher Todesfall ist entweder ein Todesfall der - plötzlich,
Abb. 1 > 3D-Dokumentation einer Bissverletzung mit Vergleich des 3D-gescannten Gebissabdrucks eines Verdächtigen. (Mit freundl. Genehmigung von $\mathrm{M}$. Thali)

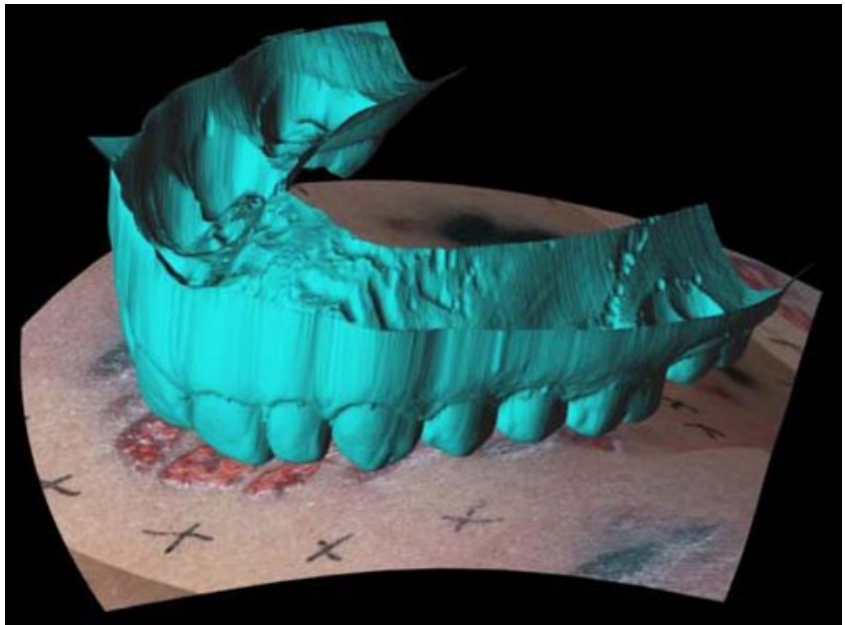



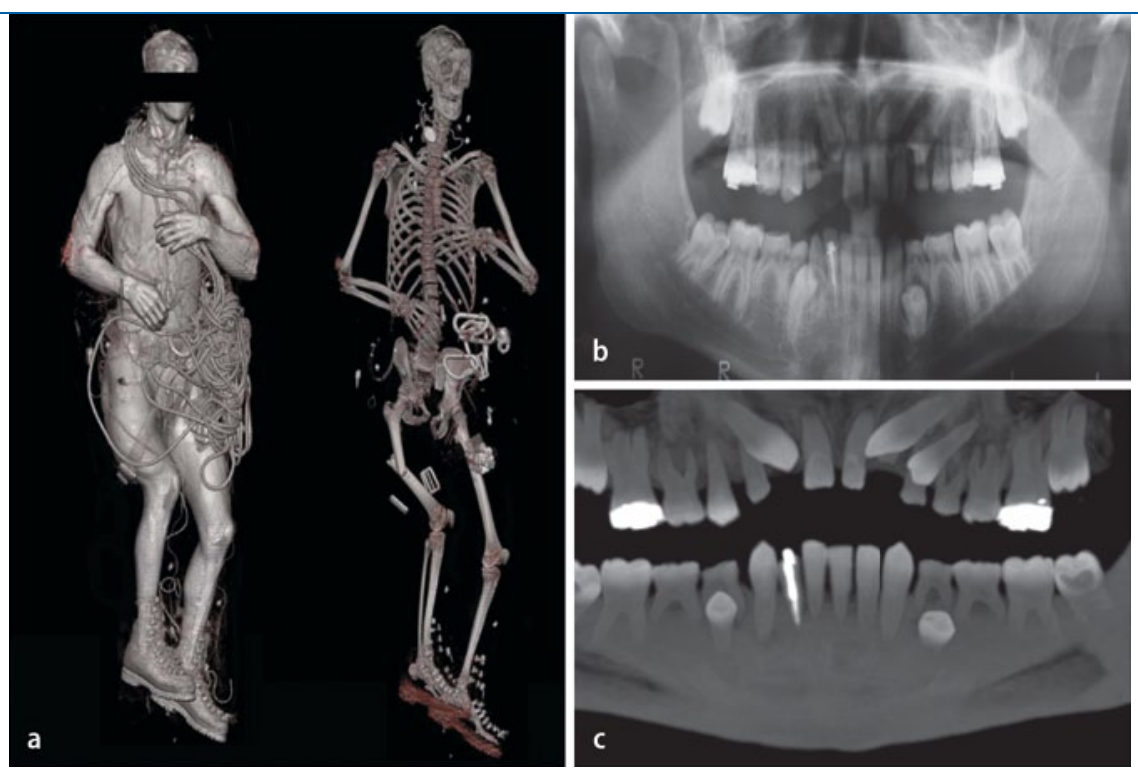

Abb. $2 \Delta$ Gesamtkörpercomputertomographie eines abgestürzten Bergsteigers (a) inklusive antemortaler (b) und postmortaler (c) radiologischer Gebissdarstellung. (Mit freundl. Genehmigung von M. Thali)
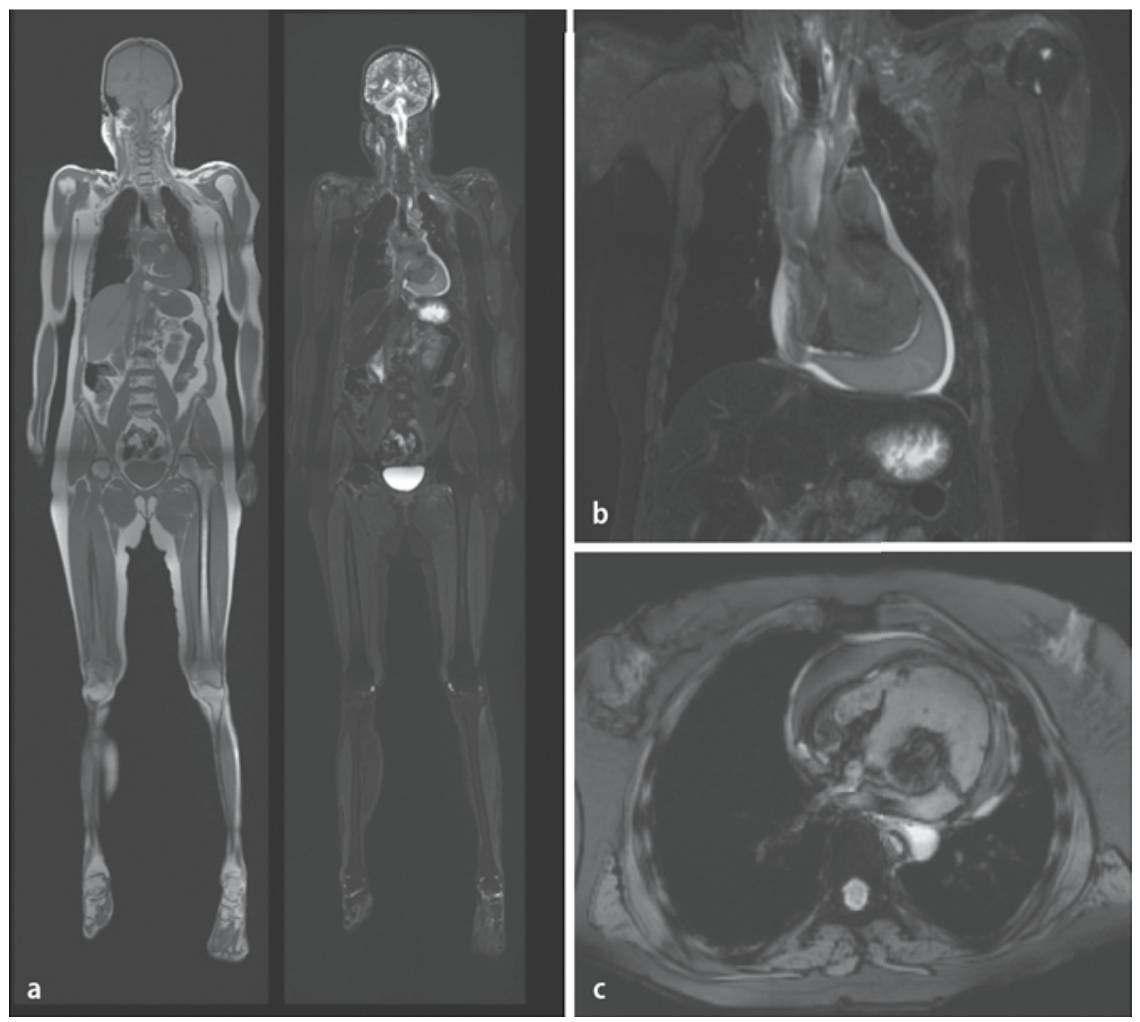

Abb. $3 \Delta$ Gesamtkörpermagnetresonanztomographie (a) mit fokussierter Darstellung einer Herzwandruptur mit Herzbeuteltamponade (b,c). (Mit freundl. Genehmigung von M. Thali)

- unerwartet oder

- auf Gewalt verdächtigt ist, inklusive der Spätfolgen davon.

Bei solchen Todesfällen besteht in der Schweiz Meldepflicht an die Untersuchungsbehörden. Man geht davon aus,
Zusammenfassung • Abstract

Pathologe 2011 - [Suppl 2] 32:292-295

DOI 10.1007/s00292-011-1520-5

C) Der Verfasser 2011

\section{Thali}

Virtuelle Autopsie (Virtopsy) in der Forensik.

\section{Vom Skalpell zum Scanner}

\section{Zusammenfassung}

"Virtopsy" nutzt moderne Imaging-Technologie, um die klassische Dokumentation der Autopsie zu optimieren. Vorteile der Virtopsy sind die untersucherunabhängige, objektive 3D-Dokumentation von Körperbefunden und der nichtinvasive Ansatz. Virtopsy ist eine Option bei Autopsieablehnung durch die Familie oder aus religiösen Gründen. Virtopsy eignet sich bei Massenkatastrophen für eine schnelle Identifizierung von Opfern. Für Staatsanwälte und Gerichte können medizinische Befunde dreidimensional ohne blutige Bilder gezeigt werden.

\section{Schlüsselwörter}

Autopsie · Virtuelle Autopsie · Rechtsmedizin . Radiologie $\cdot$ Bildgebung

\section{Virtual autopsy (virtopsy) in forensic science.} From the scalpel to the scanner

\section{Abstract}

The aim of "virtopsy" is to utilize modern imaging technology to optimize classical autopsy documentation. The benefits of virtopsy include examiner-independent, objective $3 \mathrm{D}$ documentation and its non-invasive approach. Virtopsy is an option in cases where autopsy is rejected by family members or for religious reasons. It is also suitable as a rapid identification and examination tool in largescale disasters. Forensic findings can be presented to prosecutors and courts in $3 \mathrm{D}$ and without bloody images.

\section{Keywords}

Autopsy - Virtopsy · Forensic medicine . Radiology $\cdot$ Imaging 


\section{Hauptreferate: Obduktionspathologie}
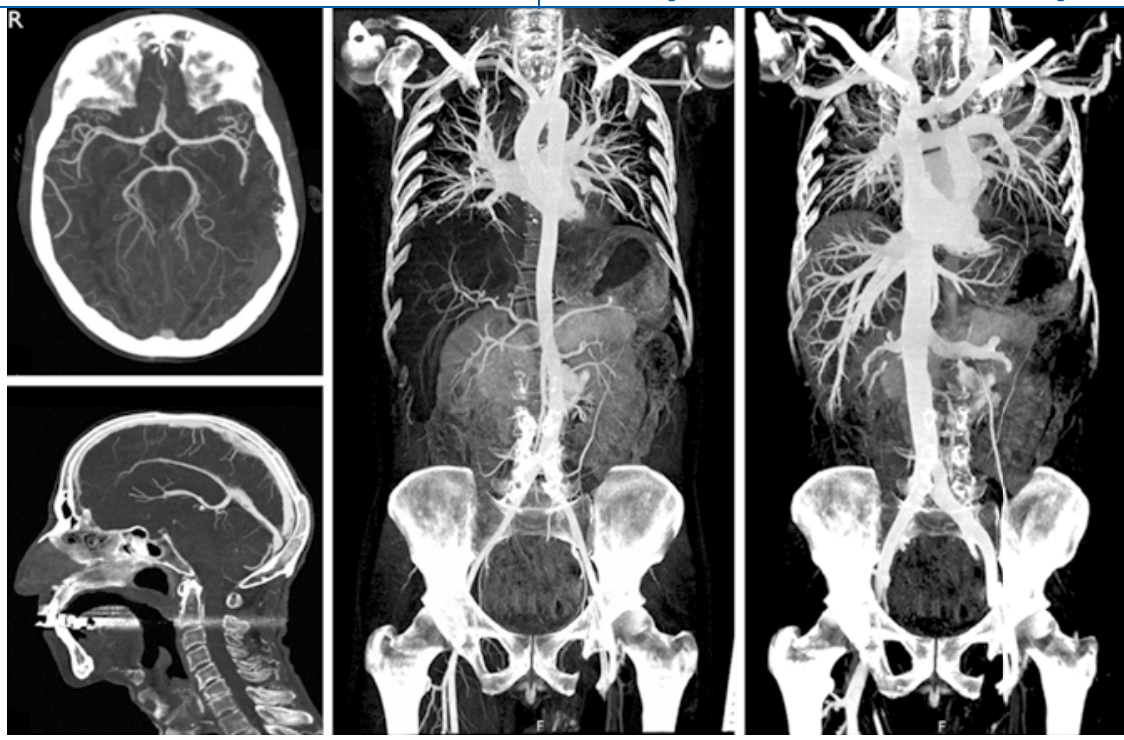

Abb. 4 \ Postmortale Gesamtkörperangiographie mit Visualisierung des Herz-Kreislauf-Systems. (Mit freundl. Genehmigung von M. Thali)

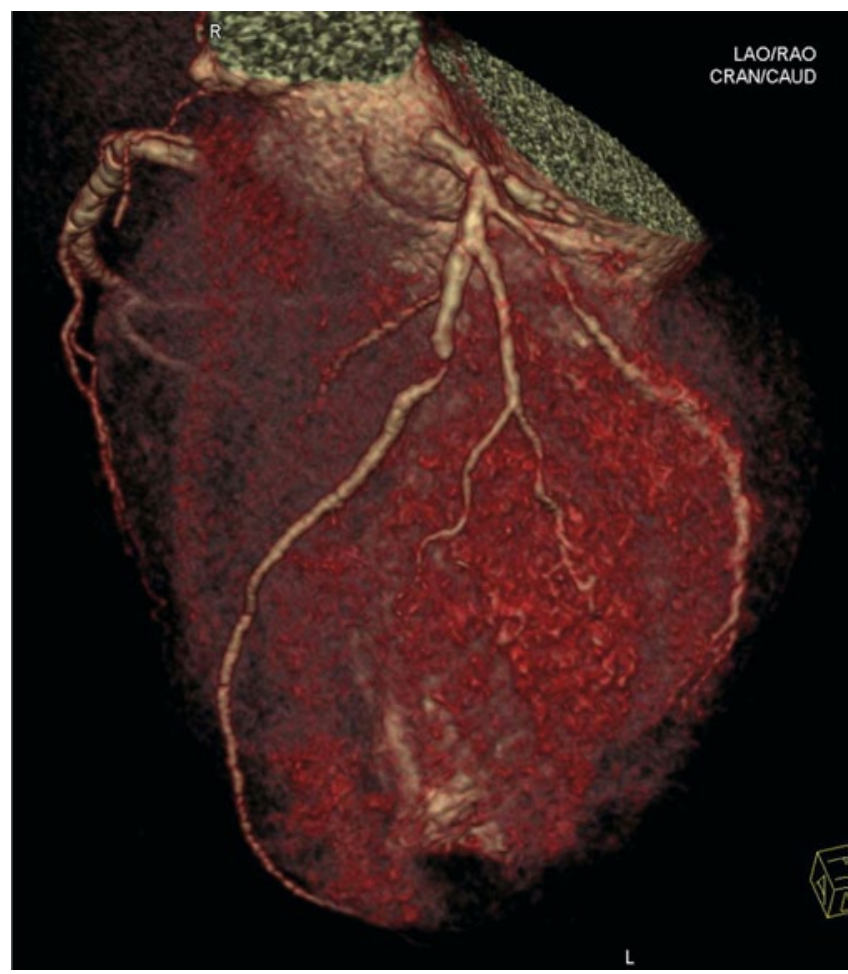

Abb. $5<$ Visualisierung einer 3D-postmortalen Angiographie mit Darstellung von stenotischen Bereichen der Herzschlagader. (Mit freundl. Genehmigung von M. Thali)

in der Klärung der Fallumstände durch die Untersuchungsbehörden.

\section{Was sind die maßgeblichen \\ Entwicklungen der Gerichtsmedizin in den letzten Jahrzehnten?}

Dies ist sicher die Verwendung bildgebender Methoden. Bildgebende Methoden wurden seit jeher in der Rechtsmedizin/Forensik verwendet, aber nicht in dem Ausmaß und der Komplexität, wie dies in der Schweiz - ausgehend vom Berner Institut - forciert wurde, und zwar in einer außerordentlichen Tiefe und Breite, die letztendlich dazu führte, dass die "Virtopsy" heute weltweit in vielen rechtsmedizinischen Instituten angewandt wird. Doch wie kam dies?

Aufgrund eines Falles musste sich das Berner Institut Mitte der 1990er-Jahre überlegen, wie man künftig sogenannte geformte, dreidimensionale Verletzun- gen an der Körperoberfläche dokumentieren will. Mit einer transdisziplinären Forschungsgruppe begann man, Technologien zu evaluieren und zu validieren, welche primär in der Forensik praktisch keine oder gar keine Anwendung fanden. Für die Körperoberflächendokumentation wurde das sogenannte photogrammetrisch gestützte $3 \mathrm{D}$-Oberflächenscanning für die Forensik weiterentwickelt und optimiert. Durch diese Technologien ist es möglich, an Lebenden und Verstorbenen geformte Verletzungen - also Verletzungen, bei denen es möglich ist, sie einem Werkzeug zuzuordnen - auch dreidimensional zu dokumentieren. Die dreidimensionale Körperoberfläche kann man somit auch dreidimensional speichern und später kann eine Match-Analyse in $3 \mathrm{D}$ durchgeführt werden (• Abb. 1). Bisher wurden $3 \mathrm{D}$-Körperoberflächenbefunde mittels Fotografie auf eine $2 \mathrm{D}$-Fotoebene reduziert.

In Ergänzung der Körperoberflächendokumentation wurden auch moderne, bildgebende Methoden wie Computerund Magnetresonanztomographie eingeführt. Ähnlich wie in der Klinik kommen solche Methoden auch in der Forensik zur Anwendung. Zusätzlich zu diesen bildgebenden Methoden wurden auch postmortale Angiographie und postmortale Biopsie eingeführt [1-9]. Alle diese Technologien werden heute robotorgestützt in den sogenannten Virtobot zusammengefasst. Mittels dieser Techniken ist es heute möglich, $60-80 \%$ der forensisch relevanten Todesursachen zu detektieren. Dies ist selbstverständlich im Bereich der klassischen pathologischen Fragestellung in diesem Ausmaß noch nicht möglich.

Die Dokumentation der Körperoberfläche und des Körperinneren mit modernen, bildgebenden Methoden macht es möglich, den Körper - der ja während einer Autopsie teilweise bezüglich der dreidimensionalen Struktur zerstört wird - zu dokumentieren und für spätere Untersuchungen zugänglich zu machen (• Abb. 2-5). Wenn ergänzend dazu auch die Umgebungssituation eines Ereignisortes dreidimensional von der Polizei gescannt wird, kann so der Ort mit den involvierten Körpern und Gegenständen dreidimensional archiviert werden (z. B. Verkehrsunfallanalysen). Tat- und Ereig- 
nisrekonstruktionen können zu einem späteren Zeitpunkt auf dem Bildschirm dreidimensional analysiert und nachvollzogen werden.

\section{Virtopsy - die Vorteile}

Vorteile der Virtopsy sind insbesondere die objektive untersuchungsunabhängige Dokumentation und die nicht destruktive minimal-invasive, maßstabgetreue 3D-Dokumentation und 3D-Rekonstruktionsoption. Sie spielt eine Rolle bei Autopsieablehnung wegen familiärer Einwände oder aus religiösen Gründen. Bei ABCTerrorismus und Massenkatastrophen dient die moderne Bildgebung der schnelleren Identifikation. Zudem ist eine Teleforensik-Zweitmeinung möglich und für die Staatsanwaltschaft und Gerichte ist die Nachvollziehbarkeit dank 3D-Visualisierung der medizinischen Inhalte ohne blutige Bilder wesentlich optimiert. Die optimierte Dokumentation mit obigen Vorzügen legitimiert in vielen Fällen die höheren Kosten der Virtopsy.

Für die neue Generation der Rechtsmediziner, welche manchmal auch „Virtopsianer" genannt werden, fließen verschiedene Gebiete wie klassische Rechtsmedizin, Radiologie und Ingenieurwesen zusammen. Dies gibt dem Nachwuchs auch neue Chancen bezüglich Forschung und Dienstleistung. Die Virtopsy wird die klassische Autopsie sicher vorerst nicht in allen Belangen ersetzen, stellt aber einen Mehrwert bzw. eine Qualitätssteigerung dar.

\section{Fazit für die Praxis}

Die Virtopsy wird die klassische Autopsie in den nächsten Jahren in der Forensik sicherlich nicht vollumfänglich ersetzen. Sie stellt aber eine Art ",forensische CTLeichenschau"-Option für außergewöhnliche Todesfälle - sozusagen als Zwischenstufe zwischen äußerer Leichenbesichtigung und klassischer Autopsie - dar. Die Virtopsy stellt für das klassische Fach Rechtsmedizin und die Staatsanwaltschaft eine neue visuelle und gutachterliche Dimension dar. Für die Nachwuchskräfte im Fach ist sie eine enorme akademische Chance. Und wie sich die Zukunft entwickeln wird, werden wir in einigen Jahren sehen.

\section{Korrespondenzadresse}

Prof. Dr. M. Thali

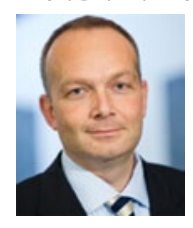

Institut für Rechtsmedizin, Universität Zürich

Winterthurerstr. 190/52

8057 Zürich

Schweiz

thali@irm.uzh.ch

Interessenkonflikt. Der korrespondierende Autor gibt an, dass kein Interessenkonflikt besteht.

The supplement this article is part of is not sponsored by the industry.

\section{Literatur}

1. http://www.virtopsy.com

2. http://www.irm.uzh.ch

3. Michael J, Thali MD, Viner Mark D, Brogdon BG (2010) Brogdon's Forensic Radiology, 2. Aufl. CRC Taylor \& Francis

4. Thali M, Dirnhofer R, Vock P (2010) The virtopsy approach: $3 \mathrm{D}$ optical and radiological scanning and reconstruction in forensic medicine. CRC Taylor \& Francis

5. Ebert LC, Ptacek W, Naether S et al (2010) Virtobot - a multi-functional robotic system for 3D surface scanning and automatic post mortem biopsy. Int J Med Robot 6(1):18-27

6. Bolliger SA, Thali MJ, Ross S et al (2008) Virtual autopsy using imaging: bridging radiologic and forensic sciences. A review of the Virtopsy and similar projects. Eur Radiol 18(2):273-282

7. Thali MJ, Jackowski C, Oesterhelweg L et al (2007) VIRTOPSY - the Swiss virtual autopsy approach. Leg Med (Tokyo) 9(2):100-104

8. Dirnhofer R, Jackowski C, Vock P et al (2006) VIRTOPSY: minimally invasive, imaging-guided virtual autopsy. Radiographics 26(5):1305-1333

9. Thali MJ, Yen K, Schweitzer W et al (2003) Virtopsy, a new imaging horizon in forensic pathology: virtual autopsy by postmortem multislice computed tomography (MSCT) and magnetic resonance imaging (MRI) - a feasibility study. J Forensic Sci 48(2):386-403 